\title{
Sistemas de producción del cacao vs recursos ambientales. Un reto estratégico actual
}

\author{
Cocoa production systems vs environmental resources. A current strategic challenge \\ Emma Yolanda Mendoza Vargas' (iD (8) , Ximena Paola Cervantes Molina² (id (8), \\ Gregorio Humberto Vásconez Montúfar ${ }^{3}$ (D) (8)
}

\section{Cómo citar:}

Mendoza, E.; Cervantes, X. y Vásconez, G. (2021). Sistemas de producción del cacao vs recursos ambientales. Un reto estratégico actual. Socialium, 5(2), 335-348. https://doi.org/10.26490/uncp.sl.2021.5.2.1009

${ }^{1}$ Magister en Dirección de Empresas, Ingeniera en Marketing.

Universidad Técnica Estatal de Quevedo,

Quevedo, Ecuador.

emendoza@uteq.edu.ec

2 Magister en Desarrollo y

Medio Ambiente, Ingeniera en Administración Financiera.

Universidad Técnica Estatal de Quevedo, Quevedo, Ecuador. xcervantes@uteq.edu.ec

${ }^{3}$ Doctor en Ciencia Agrarias, Ingeniero agropecuario.

Universidad Técnica Estatal de Quevedo, Quevedo, Ecuador. gvasconez@uteq.edu.ec

Arbitrado por pares ciegos Recibido: 10/12/2020 Aceptado: 10/06/2021

\section{RESUMEN}

Si bien, en los últimos años se ha generalizado el empleo de prácticas sostenibles, persisten una gama de efectos ambientales y sociales adversos que amenazan con revertir el desenvolvimiento productivo y económico alcanzado. Esta investigación tiene como objetivo realizar un análisis respecto a los retos medioambientales de los sistemas de producción sostenible del cacao. A partir de la revisión documental y la indagación de la literatura se pudo determinar que, a nivel mundial la producción del cacao requiere del empleo racional de recursos ambientales, ya que la contaminación y los desechos crecen. Además, la demanda de sus derivados se ha enfocado en el consumo de una oferta más amigable con el medio ambiente. Por lo tanto, se ha destacado en la literatura y desde organismos multilaterales que crear sistemas de producción más eficientes constituye una necesidad urgente. Ante estas recomendaciones, el propósito de este estudio es ofrecer una visión reflexiva y panorámica sobre los sistemas de producción del cacao y su impacto en los recursos ambientales, su implicación, consecuencias, riesgos, y el reto que representa un cambio en esta dinámica. La importancia de implementar un sistema de gestión productiva que destaque por su sostenibilidad económica, ecológica y social es un desafío clave que se inscribe entre las conclusiones obtenidas con la reflexión teórica presentada sobre los sistemas de producción del cacao y los recursos ambientales.

Palabras clave: sistemas de producción; cacao; recursos ambientales; sostenibilidad; producción agrícola.

\section{ABSTRACT}

Although the use of sustainable practices has become widespread in recent years, a range of adverse environmental and social effects persist that threaten to reverse the productive and economic development achieved. This research aims to carry out an analysis regarding the environmental challenges of sustainable cocoa production systems. From the documentary review and the investigation of the literature, it was possible to determine that, at the global level, cocoa production requires the rational use of environmental resources, since pollution and wastes grow. In addition, the demand for its derivatives has focused on the consumption of a more environmentally friendly offer. Therefore, it has been emphasized in the literature and from multilateral organizations that creating more efficient production systems is an urgent need. Given these recommendations, the purpose of this study is to offer a reflective and panoramic view on cocoa production systems and their impact on environmental resources, their implication, consequences, risks, and the challenge that represents a change in this dynamic. The importance of implementing a productive management system that stands out for its economic, ecological and social sustainability is a key challenge that is part of the conclusions obtained with the theoretical reflection presented on cocoa production systems and environmental resources.

Keywords: production systems; cocoa; environmental resources; sustainability; agricultural production. 


\section{Introducción}

La gestión sostenible y eficiente de los recursos en todas las etapas de las cadenas de valor de los bienes y servicios es de vital importancia. La promoción y creación de procesos de producción que utilicen menos recursos y minimicen el vertimiento de desechos, que produzcan beneficios ambientales y aporten económica y socialmente es una cuestión de primer orden en la actualidad (El Colegio de la Frontera Sur, ECOSUR, 2020). Estas mejoras, orientadas al aumento de la competitividad de las empresas, deben lograr que los retos de sostenibilidad se conviertan en oportunidades de negocio, empleo y exportación.

En el caso de los sistemas de producción del cacao, las premisas respecto al impacto ambiental y la eficiencia no son diferentes de las que rigen en los sistemas de producción agrícola de manera general. Ante la necesidad de obtener un mayor margen de utilidad se establece como máxima que debe imperar la sostenibilidad y no solo las estrategias enfocadas en los rendimientos. No obstante, aunque se brinda capacitación técnica a los productores, todavía persisten problemas en el uso de los recursos que desencadenan afectaciones ambientales (Arvelo et al., 2016).

La literatura previa ha identificado que el cultivo del cacao es amigable para el medio ambiente, debido a que puede combinarse con otros de ciclo corto, no exige de altos niveles de insumos externos y se convierte en hábitat de especies en zonas con afectaciones en los ecosistemas por la práctica de otras actividades productivas (Rojas y Sacristán, 2013). A pesar de ello, sus sistemas de producción no se encuentran ajenos a procedimientos de manejo inadecuados que comprometen la sostenibilidad ecológica, social y económica (Larrea, 2008).

De acuerdo con Acebo et al. (2016), la actividad cacaotera se encuentra marcada por implicaciones sociales y ambientales de importancia en un momento donde se exige la eficiencia de sus operaciones. La respuesta a esta demanda constituye un punto crítico en los procesos productivos de este subsector debido a que todavía no se ha generalizado una oferta de mayor calidad y más amigable. La interdependencia de diversos actores en la cadena de valor del grano de cacao y sus derivados ha impulsado el surgimiento de iniciativas de certificación y la implementación de sistemas de trazabilidad, pero aún a muy baja escala.

Si bien esta relación entre los sistemas de producción del cacao y los impactos ecológicos no es un tema de preocupación reciente, aún no se alcanza la cultura necesaria para minimizar los grandes costos humanos y económicos a consecuencia de la contaminación y la degradación de recursos ambientales. Este panorama, con variaciones entre los países, ha demostrado que la dificultad está en sostener un equilibrio entre la conservación del medio, su calidad, y las consecuencias procedentes de una mayor producción, a partir de los sistemas existentes (Arvelo et al., 2016). La mayoría de la producción más allá 
de las pequeñas y medianas fincas considera económicamente rentable el uso intensivo de fertilizantes, minerales y plaguicidas, y no ha habido una aceptación general de tecnologías de producción más limpias como el sistema integrado de nutrición de plantas y la revisión integral de las plagas (Organización de las Naciones Unidas para la Educación, la Ciencia y la Cultura, UNESCO, 2019).

Ante estas breves puntualizaciones sobre el panorama actual de los sistemas de producción y los recursos ambientales, es de interés en este artículo centrarse específicamente en la producción de cacao y en su impacto medioambiental, a partir de un énfasis reflexivo, desde una aproximación teórica, en los riesgos y en el reto estratégico que conlleva su dinámica. Para ello, se realiza una revisión de fuentes bibliográficas que conduce a la exposición de conceptos y criterios. Se refieren, además, breves tendencias mundiales, regionales y nacionales, así como contribuciones y afectaciones relevantes hasta nuestros días en relación con la explotación y desarrollo, no solo de los sistemas de producción del cacao, sino también de los recursos ambientales disponibles.

Esta investigación tiene como objetivo realizar un análisis respecto a los retos medioambientales de los sistemas de producción sostenible del cacao, capaz de propiciar una mayor conciencia sobre estos los recursos ambientales involucrados y la sostenibilidad de este sector de la agricultura.

\section{Método}

Tipo y diseño de estudio. Se desarrolló una exposición sintética y reflexiva sobre los sistemas de producción del cacao y el impacto que estos provocan en los recursos ambientales. Se adoptó básicamente un enfoque cualitativo, el cual, orientándose al ámbito descriptivo, permitió ofrecer una perspectiva amplia sobre la temática escogida por medio de la información existente sobre el tema en cuestión. De acuerdo con este enfoque, se consigue una caracterización del tema a lo largo del tiempo, describiéndose los elementos sobre los cuales se determina su importancia; explorando sus tendencias y cómo esto determina los retos a alcanzar en el futuro en materia de sostenibilidad ambiental en cuanto a la producción cacaotera.

Procedimientos de la recolección de datos. La información que sirvió como insumo para el desarrollo del trabajo, se la recopiló durante el primer semestre del año 2021, mediante la revisión documental y el aprovechamiento de la información existente sobre el tema, lo cuál posibilitó su análisis e interpretación, así como también la elaboración de criterios que ayudaron a fundamentar la relación entre los sistemas de producción del cacao y los recursos ambientales, variables independiente y dependiente respectivamente. 
Los métodos teóricos empleados fueron: histórico-lógico: para determinar las tendencias, el desarrollo y evolución de los sistemas de producción; análisis y síntesis: para el desarrollo de todo el proceso de investigación científica; abstracción-concreción: durante toda la investigación, fundamentalmente para inferir los elementos teóricos y concepciones; y análisis de documentos: para caracterizar los sistemas de producción del cacao y sus retos o desafíos.

Aspectos éticos. En cuanto al aspecto ético; se dio tratamiento de manera responsable a la información recopilada, respecto al derecho intelectual de cada uno de los autores citados, y a partir de esta acción se realizó el análisis, la interpretación y la elaboración de criterios para fundamentar las variables independientes y dependientes abordadas en el tema de estudio.

\section{Resultado}

\section{Sistemas de producción, recursos ambientales y enfoque sostenible}

Actualmente existe un interés marcado en la producción y comercialización del cacao, su consumo a nivel internacional es relevante, no solo como chocolate, sino también como ingrediente básico para la alimentación, dada la revaloración de la exquisitez de sus granos (Mendoza et al., 2020).

Para analizar un sistema de producción, primeramente, es necesario esclarecer que un sistema puede ser definido como un todo integrado por partes relacionadas que coexisten para conseguir un determinado objetivo. Las empresas son consideradas sistemas totalmente abiertos. Importan recursos, transforman y procesan recursos, así como también exportan el resultado de las transformaciones que realizan de regreso al ambiente por medio de sus salidas. La relación entradas/salidas muestra la eficacia del sistema (Programa de Naciones Unidas para el Medio Ambiente, PNUMA, 2020).

Un sistema de producción es el modo en que se efectúa la entrada de las materias primas, así como su proceso de transformación y obtención del producto para ofrecer a los clientes, tomando en cuenta los controles necesarios. Se ha determinado, por otra parte, la existencia de tres tipologías tradicionales de sistemas de producción: la producción por lotes; la producción continua, la producción por trabajos o bajo pedido; y una cuarta tipología llamada tecnología de grupos (ECOSUR, 2020).

En cuanto a recursos ambientales, debe considerarse que, desde el punto de vista de concepto de recurso, y dado que se clasifican en renovables y no renovables, procede de una idea moderna del vínculo hombre - sociedad - naturaleza. Estos son definidos como todos aquellos bienes de la naturaleza que utiliza el ser humano para su provecho, tales como alimentos, fuentes de energía y materias primas. Los recursos ambientales se transforman en bienes o servicios con el fin del consumo cuando pasan por procesos de extracción y transformación. Claramente, estos recursos se clasifican de acuerdo con 
criterios como: según su capacidad para renovarse; según su disponibilidad para la explotación e importancia, por lo que de ellos depende la supervivencia de la humanidad, así como el desarrollo cultural y económico de cada generación (ECOSUR, 2020).

Los modos en que son utilizados los recursos ambientales con el fin de producir alimentos penden mucho de factores económicos, humanos, sociales y culturales. Esto se evidencia en el gran número de cambios ejecutados en la naturaleza al modificar paisajes, mediante el uso y la eliminación de especies y plantas, variedades animales, manipulando las aguas y suelos, etc. El gran impacto que han producido los sistemas de producción de alimentos sobre el medio ambiente redireccionó la atención sobre la calidad del crecimiento económico a partir de los sistemas de producción, las necesidades de las generaciones presentes frente a las de generaciones futuras, las disparidades sociales, y la proporción entre los intereses de las regiones, las naciones y a nivel mundial (Martínez, 2009).

Por medio de la eficiencia en el uso de los recursos existen aún oportunidades claves para revertir esta tendencia insostenible: generar economías verdes donde el desarrollo económico se desligue del daño medioambiental. Al promoverse la producción y el diseño de bienes y servicios de bajo impacto al medio ambiente, la eficiencia en la utilización de los recursos ha de contribuir a la satisfacción de las necesidades humanas con respeto a la capacidad de carga ecológica del planeta. De acuerdo con PNUMA (2020), esta noción de eficiencia puede definirse desde la representación del ciclo de vida y de la cadena de valor, lo cual presume la reducción del impacto ambiental total del consumo y la producción de bienes y servicios, desde la extracción de las materias primas hasta su uso final.

El enfoque de desarrollo sostenible comprende dimensiones y objetivos centrales que son: el ambiental, el económico y el social. De esta forma el crecimiento económico no se enfoca solo en cuestiones de mayor producción, sino también en la generación de equidad social y sustentabilidad ambiental que se extiende a todos los sistemas de producción, como el cacao (Rojas \& Sacristán, 2013). Así se busca desarrollar la economía a partir del aprovechamiento de oportunidades sobre la inversión de tecnologías que contaminen menos. Además, promueve la creación de empleos verdes con el fin de aminorar la pobreza e impactar en el bienestar de las personas (La Organización de las Naciones Unidas para la Alimentación y la Agricultura. FAO, 2020).

\section{Sistemas de producción del cacao y recursos ambientales}

La producción de cacao implica una secuencia de procesos de manera lógica que busca desarrollar el cultivo de forma correcta desde el inicio de la actividad hasta su aprovechamiento. Principalmente, su producción no tiende a incorporar alta tecnología y por ello, sus afectaciones ambientales no sobresalen respecto a otros sistemas de producción. En cada una de las etapas del cultivo es común que se realicen las actividades de manera manual debido a la baja modernización de sus procesos. Además, la literatura 
ha reconocido que destaca por la implementación de sistemas agroforestales con beneficios para sus procesos productivos y para el ambiente.

Con la implementación de estos sistemas, se promueve el desarrollo sostenible, siempre y cuando su incorporación sea factible y viable. De acuerdo con Rojas y Sacristán (2013), los modelos agroforestales proporcionan no solo la sombra que requiere este cultivo, sino también propicia que se obtengan ingresos a partir del cultivo de otras especies. También, al vincular otros cultivos se beneficia al ambiente con el aporte de biomasa y nutrientes. Pero, el desarrollo de estos sistemas y sus resultados no se logran de manera espontánea. El cultivo del cacao bajo un sistema agroforestal exige de la consideración de cuestiones puntuales, tales como: las metas del cultivo, la idoneidad de las especies a incorporar y su distribución, el material genético, el entorno agroecológico, la demanda y la posesión o acceso a recursos.

Aunque la producción de cacao se puede identificar como poco contaminante, sus actividades generan impacto ambiental cuando no se implementan prácticas adecuadas. Por ejemplo, un sistema de riego no eficiente implica altas probabilidades de erosión del suelo y la consiguiente pérdida de fertilidad. También un problema de este tipo conlleva a que se afecten las fuentes de agua, al disminuir su caudal. Al unísono destacan los problemas que generan las fundas y recipientes, así como los procesos que se realizan luego de emplear sus contenidos o de limpiar el área luego de la cosecha.

Otros autores han reconocido que los problemas de erosión son más frecuentes en etapas puntuales del ciclo de cultivo (Ramos, 2014). Al respecto se ha señalado que los mayores impactos se presentan al momento de establecer el vivero y durante la siembra, debido a la remoción del material vegetal existente. Los procesos de afectación al suelo tienden a predominar en las áreas de laderas. En estas zonas es más frecuente el arrastre de material como consecuencia de lluvias, vientos o sistemas de riesgos ineficientes.

De conjunto con los impactos en la agricultura, el procesamiento del grano de cacao genera importantes secuelas ambientales, a través del consumo de energía y la generación de residuos. La degradación terrestre y el uso insostenible del agua también contribuyen a disminuir la capacidad de la base de recursos naturales para suministrar alimentos (PNUMA, 2020).

\section{Datos relevantes en relación con los sistemas de producción de cacao y uso de recursos ambientales}

En la actualidad se adolece de información precisa sobre el área sembrada de cacao en el mundo. No obstante, algunas estimaciones permiten conocer que no sobrepasa los siete millones de hectáreas, con un mayor predominio en África, América y Asia (Rojas y Sacristán, 2013). Aunque el consumo de los derivados de cacao es alto, los precios de este producto son muy volátiles, cambiando no solo de manera 
semanal, sino también diaria. La comercialización del commodity cacao en grano depende en gran medida de las industrias procesadoras y de la concentración de capital en las grandes compañías.

A nivel internacional se ha clasificado como bipolar la cadena de valor. La producción de derivados se encuentra en manos de cinco grandes firmas que controlan más del $50 \%$ de la producción total. También existe otro grupo conformado por tres empresas que gestionan la mitad del suministro internacional de ingredientes del cacao. Por su parte, la producción del cacao en granos se distingue por una destacada presencia de plantaciones pequeñas que generan una distribución asimétrica, donde el productor solo recibe menos del 6\% del precio final (Acebo, 2016).

Aunque los productores de fincas pequeñas se ven afectados por sus ganancias debido al incremento de los intermediarios, el cultivo en áreas de este tipo genera menos consecuencias ambientales. En estas fincas se suele invertir menos en productos químicos y los productores tienden a dedicar más tiempo al cuidado manual. Además, son más tendientes a emplear sistemas agroforestales, lo cual favorece la biodiversidad y minimiza las posibilidades de erosión del suelo (Arvelo et al., 2016).

La producción de cacao, de manera general, no se encuentra ajena a las demandas de prácticas amigables. Su implementación ha propiciado el acceso a nuevos destinos mediante el cumplimiento de procesos de certificación. Desde el año 2008, la comercialización internacional de cacao reporta un incremento en productos certificados, lo cual refleja una tendencia de mayor preferencia por una oferta que cumpla con parámetros de calidad. Por ejemplo, la cifra de este cacao aumentó de 200 mil toneladas en el 2010 a más de un millón de toneladas en 2013. Sin embargo, todavía los precios del cacao no certificado y la existencia de prácticas no leales en el acceso a mercados a través de la certificación contribuyen a que el producto de mayor calidad y bajo prácticas amigables deba venderse a un costo menor (Acebo et al., 2016).

La extensión de las áreas de cultivo y de industrias de procesamiento para satisfacer la demanda mundial ha generado afectaciones a la biodiversidad. Además, las quemas para limpiar las áreas son frecuentes en fincas pequeñas y ello ocasiona afectaciones ambientales diversas. Los consumidores cada vez son más exigentes respecto al acceso de un cacao orgánico, sin embargo, los sistemas de producción no se transforman al mismo ritmo de crecimiento de esas demandas.

\section{Producción sostenible de cacao en América Latina. Situación en Ecuador}

La producción de cacao en América Latina implica a trabajadores individuales y a familias completas. En la región destaca que cerca del $90 \%$ de la producción radica en pequeños y medios agricultores, y las fincas oscilan principalmente entre las 2 y 5 hectáreas. En los últimos años se ha reportado un aumento del área de producción y esta dinámica se ha desarrollado de manera más sostenible en comparación con las expansiones que ocurren en otras regiones del mundo (Arvelo et al., 2016). Una de las razones 
incuestionables para esta afirmación es que, este cultivo requiere mucha mano de obra y la mayoría de las prácticas realizadas son a mano (Cervantes et al., 2020). Por otro lado; a pesar de que en algunos lugares las plantaciones de cacao se aprovechan como rutas turísticas, el impacto de estos emprendimientos turísticos y productivos ha sido positivo, al repercutir de manera controlada y recuperable sobre los recursos naturales que poseen (Mendoza et al., 2020). Sin embargo, las transformaciones en beneficio del medio ambiente todavía resultan incipientes y muestra de ello es la modernización de la producción y el fomento del consumo responsable.

El manejo sostenible de los recursos ambientales y el revertir la degradación de los recursos son factores de importancia clave en los sistemas productivos cacaoteros. Ecuador ha tomado partida en este empeño. Varias han sido las acciones realizadas para habilitar al país y hacer frente a esta problemática, posicionándose como referente a nivel internacional. No obstante, los problemas relacionados a la explotación de los recursos ambientales a partir de los sistemas de producción continúan existiendo. Ante esta realidad se persigue el propósito de desvincular el desarrollo económico de la degradación ambiental al tiempo que se asegure la transición a economías verdes, generando oportunidades y bienestar para todos (Ministerio del Medio Ambiente y Ministerio de Agricultura y Ganadería de Ecuador, 2018).

Ecuador es el mayor productor mundial de cacao fino o de aroma y como sucede en la región, la mayoría de sus productores son pequeños y medianos. Aunque la producción de cacao ha desempeñado históricamente un rol de peso en la economía nacional, su valor ha disminuido respecto al inicio del siglo pasado. En los últimos años se ejecutan programas de reactivación ante el impacto de la longevidad de los cultivos, la baja productividad, los problemas con los incentivos a los productores y una extensa cadena de comercialización que afecta las ganancias de los productores (Acebo, 2016).

El cultivo del cacao nacional está supeditado al manejo dado en cada una de las fincas. De las prácticas asumidas por los agricultores depende el empleo de recursos ambientales, los beneficios y consecuencias para los ecosistemas (Larrea, 2008). A ello se agrega que, aunque existen avances en la expansión y promoción de la producción más limpia como política nacional, las orientaciones no son consecuencia de estudios de casos. En las propuestas no destacan estrategias que respondan a buenas prácticas y se han relegado los análisis y las iniciativas para sectores productivos importantes del país como las pequeñas y medianas industrias de procesamiento de cacao en comparación con otros enclaves del sistema alimentario (Ramos et al., 2020).

\section{Gestión de recursos en los sistemas de producción del cacao}

Aunque a nivel internacional se han dado cambios en la dinámica productiva del cacao, su alto consumo genera riesgos de sobreexplotación de los recursos ambientales, poniendo en peligro a diferentes 
ecosistemas. De continuar explotándose los recursos ambientales a este ritmo o generados los impactos ambientales negativos ya descritos quedarán devastados los fundamentos del desarrollo económico y las bases vitales de las generaciones futuras. Los problemas de sostenibilidad que resultan de las prácticas irracionales en esta actividad comprometerán el bienestar en otros ámbitos de la dinámica social a corto, mediano y largo plazo (PNUMA, 2010).

Ante este panorama, el desarrollo de programas diversos que incluyan ayuda técnica y capacitación es de vital importancia. Fomentar una gestión sostenible desde diversos ámbitos y mediante múltiples mecanismos favorece la identificación de potencialidades de aprovechamiento e implementación de negocios sostenibles. La creación de empleos desde esta perspectiva no afecta el consumo de los derivados del cacao y, además, promueve la unificación de las personas a un sistema económico local, que genera bienestar sin necesidad de sobreexplotar los recursos ambientales.

Los sistemas de producción de cacao requieren de equilibrios entre las nuevas y viejas áreas sembradas, entre las nuevas demandas del consumidor y las condiciones de producción y en la generalización de prácticas más sostenibles. Esta última premisa de la gestión implica imbricar al sector en los proyectos nacionales de desarrollo sostenible. Como consecuencia deben surgir programas y estrategias previamente planificadas que a mediano plazo contribuyan al intercambio y trabajo conjunto regional. El punto inicial de toda transformación se encuentra en progresar respecto al conocimiento y la información de los involucrados.

Aunque una gestión sostenible de los sistemas de producción cacaoteros es compleja, Rojas y Sacristán (2013) aseguran que se puede lograr cuando se integra voluntad individual y colectiva, cuando se involucra a decisores y cuando se ponen a disposición del cambio recursos materiales de manera sostenida. Algunos de las transformaciones en las fincas implican la extensión de modelos agroforestales que tienen un impacto integral cuando su proceso de implementación también se encuentra guiado por decisiones sostenibles.

Ante el desarrollo actual y las exigencias de ciudadanos un poco más conscientes del cuidado ambiental, surgen oportunidades comerciales para los productores y comercializadores del grano, así como para los elaboradores de sus derivados que exigen de una mayor calidad en los productos obtenidos a través de procesos sostenibles social y ambientalmente. Una de las cuestiones de la realidad del cacao que limitan ese propósito es su base genética. Actualmente se requiere de una mayor diversidad para generar variedades mejoradas y ello se encuentra directamente relacionado con una transformación en aquellas prácticas que culminan en la destrucción de bosques tropicales, en el cambio climático y en la mayor frecuencia de desastres naturales (Arvelo et al., 2016). 


\section{Discusión}

El crecimiento económico conseguido hasta el momento a costa del planeta será insostenible mantenerlo a futuro (PNUMA, 2020). Los sistemas de producción de cacao en relación con los recursos ambientales no se encuentran ajenos a esas alertas de quiebre que ofrece el planeta. Por lo tanto, se requiere de la generalización de prácticas más sostenibles a nivel micro, meso y macro. Por ejemplo, se ha destacado que los productores de chocolate Premium necesitan de una mayor estabilidad y de estándares de eficiencia en los flujos del grano. Ello implica acercar el cultivo y procesamiento del cacao a la innovación, con la finalidad de que se pueda identificar el trayecto de los insumos, la marca de carbono que contiene una barra de chocolate y la reducción de los riesgos sociales que implica su producción y que en muchos casos se encuentran asociados a la explotación de la fuerza laboral y al trabajo infantil.

El mayor reto de la industria de cacao radica en la sostenibilidad del suministro. Estudios recientes han demostrado que crece la práctica entre los consumidores de conocer sobre el contenido y la seguridad de los productos, lo cual implica ofrecer datos cada vez más objetivos y confiables sobre la dinámica productiva y el origen de las materias primas. De ahí que, en estos momentos, se requiera de la implementación de procesos de mayor transparencia y sistemas de trazabilidad que no solo incluyan los aspectos más cercanos a la obtención del producto final, sino también la procedencia del grano de cacao, las condiciones de cultivo y su impacto en el medio ambiente (Acebo et al., 2016).

La disponibilidad de recursos como tierra y agua para la agricultura compite con otros proyectos económicos y sociales. Ante este escenario, se ha demostrado que la extensión de la frontera agrícola afecta a los hábitats naturales y se requiere de una mayor productividad para que el cultivo de alimentos, como el cacao, no sea producto de prácticas irracionales, sino que regida por la eficiencia y la sostenibilidad se convierta en generador de externalidades positivas. Avanzar en ese sentido implica reforzar las instituciones de control y ajustar las normas de producción a las exigencias medioambientales de estos tiempos.

Los procesos de innovación no pueden distanciarse del desarrollo tecnológico en un sistema productivo cacaotero que es asimétrico y que destaca por la concentración en los eslabones finales de la cadena productiva, mientras que la base se compone de pequeñas fincas. La expansión de la tecnología no solo se relaciona con la maquinaria, sino también con los canales de venta, la promoción y enseñanza de las actividades relacionadas con el cultivo y el procesamiento del cacao (Arvelo et al., 2016).

Este cambio en la tecnología e innovación no se puede lograr sin ayuda externa, al considerar que el 90\% de los productores de cacao son pequeños y viven en condiciones de pobreza (Arvelo et al., 2016). Como su economía depende de esta actividad, realizar transformaciones resulta más costoso en un contexto 
donde destaca la baja productividad por el rezago tecnológico, las barreras para acceder a insumos y servicios y un sistema productivo dependiente. Generar transformaciones a futuro implica tener presente estas realidades actuales que implican apoyos externos para lograr un cambio no solo ambiental, sino también social.

A todo lo anterior, se suman desafíos que son consecuencias de fenómenos más actuales, con base en prácticas irracionales. Por ejemplo, el impacto de la COVID-19 puede valer de catalizador para cambios en relación con empleo de los recursos naturales. Es apremiante encontrar nuevas soluciones que prometan modalidades de producción; se identifique en la cadena de valor los puntos críticos, se comprendan mejor los efectos en el medio ambiente de los productos y servicios, y se realicen intervenciones de mayor potencial. Además, es inaplazable que los sistemas de producción se vinculen a innovación para trazar soluciones que puedan motivar a las personas a llevar estilos de vida más sostenibles, disminuyendo los efectos y aumentando las posibilidades de bienestar.

\section{Conclusión}

La presentación de algunos problemas sobre los sistemas de producción del cacao y los recursos ambientales, así como los retos y la urgencia de cambiar pautas respecto a la producción y el consumo, ha sido posible gracias al análisis de diversas publicaciones científicas que giran sobre el tema central. De acuerdo con la discusión realizada pueden señalarse las siguientes conclusiones:

El enfoque de desarrollo sostenible comprende dimensiones y objetivos centrales que son: el ambiental, el económico y el social. De esta forma el crecimiento económico no se enfoca solo en cuestiones de mayor producción, sino también en la generación de equidad social y sustentabilidad ambiental que se extiende a todos los sistemas de producción, como el cacao. Aprovechar eficientemente los recursos naturales no sólo es un factor de competitividad y costo decisivo para los sistemas de producción, sino que contribuye a la conservación de recursos ambientales a largo plazo.

La cadena de valor del cacao es bipolar. La producción de derivados se encuentra en manos de cinco grandes firmas que controlan la producción total en su mayoría. También existe otro grupo conformado por tres empresas que gestionan la mitad del suministro internacional de ingredientes del cacao. En el caso del cultivo del cacao en granos destaca la presencia de plantaciones pequeñas que generan una distribución asimétrica, donde el productor recibe un bajísimo porcentaje del precio final.

Los consumidores cada vez son más exigentes respecto al acceso a un cacao orgánico, sin embargo, los sistemas de producción no se transforman al mismo ritmo de crecimiento de esas demandas. Aunque la producción de cacao se puede identificar como poco contaminante debido al predominio de pequeñas y 
medianas fincas y la baja modernización de los sistemas productivos, procedimientos inadecuados en el cultivo y el procesamiento del grano de cacao generan afectaciones ambientales importantes, principalmente al suelo, al aire, a la vegetación y al agua.

El reto fundamental se basa en gestionar de manera más eficiente los sistemas de producción de cacao. El impacto condicionará mejores condiciones de vida y reducción de la pobreza, a partir de una gestión sostenible de los recursos ambientales. Se requieren estrategias innovadoras, de la expansión de una producción más limpia, de un reforzamiento en las instituciones de control y de la implementación de sistemas de trazabilidad. Por lo tanto, un cambio en el sistema de producción del cacao y los recursos ambientales implica aportes de cada uno de los involucrados a nivel micro, meso y macro.

\section{Referencias}

Alcívar, C., Calderón, J. y Salazar, K. (2015). La ruta del cacao, modelo de aporte al desarrollo socioturístico y económico de las comunidades ecuatorianas. Revista Observatorio de la Economía Latinoamericana, 1 - 17. https://www.eumed. net/ cursecon/ ecolat/ec/2015/cacao.html.

Acebo, M., Rodríguez, J. y Quijano J. (2016). Estudios Industriales. Orientación estratégica para la toma de decisiones: Industria del Cacao. ESPAE. http://infocafes.com/portal/biblioteca/estudiosindustriales-orientacion-estrategica-para-la-toma-de-decisiones-industria-de-cacao/.

Arvelo, M., González, D., Delgado, T. y Maroto, S. (2016). Estado actual sobre la producción, el comercio y el cultivo del cacao en América Latina. Instituto Interamericano de Cooperación para la Agricultura. http://repositorio.iica.int/bitstream/handle/11324/2793/BVE17048806e.pdf.

El Colegio de la Frontera Sur. (2020). Sistemas de producción y manejo de recursos naturales. http://www.ecosur.mx/sitios/catalogo_pub/category/sistemas-de-produccion-y-manejo-derecursos-naturales.

Cervantes, X., Mendoza, E., Vásconez, G. y Samaniego, E. (2020). Bibliometric visualization in the area of cocoa cultivation evaluating administrative and socioeconomic competencies at the Latin American leve. Revista Journal of Alternative Perspectives in the Social Sciences, 10 (4), 12891302. https://uploads.documents.cimpress.io/v1/uploads/e1cb50e5-05e8-440d-b03a071c0281468 $\sim 110$ /original?tenant=vbu-digital.

Larrea, M. (2008). El cultivo de Cacao Nacional: un bosque generoso: Manual de campo para la implementación de prácticas amigables con la biodiversidad en cultivos de Cacao Nacional. EcoCiencia / CORPEI. https://biblio.flacsoandes.edu.ec/libros/digital/43804.pdf.

Martínez-Castillo, R. (2009). Sistemas de producción agrícola sostenible. Tecnología en Marcha, 22(2), 23-39. https://revistas.tec.ac.cr/index.php/tec_marcha/article/view/114. 
PROAmazonía (2018). Programa Integral Amazónico de Conservación de Bosques y Producción Sostenible. Ministerio del Medio Ambiente de Ecuador; Ministerio de Agricultura y Ganadería. https://www.proamazonia.org/.

Mendoza, E., Cervantes, X. y Vásconez, G. (2020).The Cacao Routes as a tourist attraction in Ecuador and its impact on the communities in which they are located. Journal of Alternative Perspectives in the Social Sciences, 10 (4), 1253-1272.

https://uploads.documents.cimpress.io/v1/uploads/21623f1c-5f93-49f6-a57f06934c2af48a 110/original?tenant=vbu-digital

Mendoza, E., Boza J. y Rodríguez (2020). Las rutas del cacao y su impacto socioeconómico en las comunidades. Revista Centro Sur, 68-77. http://www.centrosureditorial.com/index. $\mathrm{php/revista/article/view/90/254.}$

Organización de las Naciones Unidas para la Alimentación y la Agricultura (1996). Cumbre Mundial sobre la Alimentación. http://www.fao.org/wfs/index_es.htm.

Organización de las Naciones Unidas para la Educación, la Ciencia y la Cultura (2019). Informe Mundial de las Naciones Unidas sobre el Desarrollo de los Recursos Hídricos. https://es.unesco.org/watersecurity/wwap/wwdr/201.

Organización de las Naciones Unidas para la Alimentación y la Agricultura (2020). El estado mundial de la agricultura y la alimentación. http://www.fao.org/publications/sofa/es/.

Programa de las Naciones Unidas para el Medio Ambiente (2010). Perspectiva Global sobre Políticas de Consumo y Producción Sostenibles. http://www.prodetur.es > prodetur > AlfrescoFileT.

Programa de Naciones Unidas para el Medio Ambiente. (2020). Eficiencia en el usode los recursos. http://www.pnuma.org > biblioteca_virtual > Web.

Ramos, D. (2014). Consideraciones ambientales y viabilidad socioeconómica del sistema productivo del cacao. [Trabajo de grado. Pontificia Universidad Javeriana]. https://1library.co/document/eqo3585q-consideraciones-ambientales-viabilidad-socioeconomicasistema-productivo-agricultores-departamento.html.

Ramos, T., Guevara, D., Sarduy, L., y Diéguez, K. (2020). Producción más limpia y ecoeficiancia en el procesado de cacao: un caso de estudio de Ecuador. Revista Investigación y Desarrollo, 20(1), 135-146. http://www.scielo.org.bo/scielo.php?pid=S251844312020000100010\&script=sci_abstract.

Rojas, F. y Sacristán, E. (2013). Guía Ambiental para el cultivo del cacao. Bogotá: Federación Nacional de Cacaoteros.

https://www.fedecacao.com.co/site/images/recourses/pub_doctecnicos/fedecacao-pubdoc_05B.pdf. 


\section{Contribución de los autores}

EYMV: Conceptualización, metodología, investigación, redacción: borrador original.

XPCM: Metodología, validación, redacción: borrador original.

GHVM: Metodología, validación, redacción: revisión y edición.

\section{Fuentes de financiamiento}

La investigación fue realizada con recursos propios.

Conflictos de interés

No presenta conflicto de intereses.

\section{Correspondencia}

emendoza@uteq.edu.ec 